\title{
Gadolinium Deposition in Neurology Clinical Practice
}

\author{
Tyler E. Smith, MD, ${ }^{1}$ Andrew Steven, MD, ${ }^{2,3}$ Bridget A. Bagert, MD, MPH $^{1,3}$ \\ ${ }^{1}$ Department of Neurology, Ochsner Clinic Foundation, New Orleans, LA ²Department of Radiology, Ochsner Clinic Foundation, New \\ Orleans, LA ${ }^{3}$ The University of Queensland Faculty of Medicine, Ochsner Clinical School, New Orleans, LA
}

\begin{abstract}
Background: Magnetic resonance imaging (MRI) enhanced with gadolinium-based contrast agents (GBCAs) is an essential tool in the diagnosis and management of many neurologic diseases, including multiple sclerosis, brain tumors, and infections. The clinical utility of GBCAs is evidenced by their widespread use. GBCAs are produced in macrocyclic and linear forms. Since 2014 , evidence has suggested that repeated administration of GBCAs can lead to gadolinium deposition in the brain.

Methods: We review the literature on gadolinium deposition, including both animal and human studies, as well as the literature on GBCA-associated health outcomes. Additionally, we summarize and discuss the updated medical society recommendations and perspectives on GBCA use in clinical practice.

Results: The first publication reporting gadolinium deposition in the human brain was published in 2014. Since that seminal report, multiple studies have demonstrated that exposure to linear GBCAs is associated with gadolinium deposition in the dentate nucleus and globus pallidus as seen on brain MRI. Macrocyclic GBCA exposure has not convincingly been associated with gadolinium deposition evident on brain MRI.

Conclusion: Clear evidence demonstrates that GBCAs lead to gadolinium deposition in the brain in a dose-dependent manner; however, only linear GBCAs have been associated with gadolinium deposition visualized on MRI. To date, no evidence links gadolinium deposition with any adverse health outcome. Updated medical society guidelines emphasize the importance of an individualized risk-benefit analysis with each administration of GBCAs.
\end{abstract}

Keywords: Contrast media, gadolinium, magnetic resonance imaging

Address correspondence to Bridget A. Bagert, MD, MPH, Department of Neurology, Ochsner Clinic Foundation, 1514 Jefferson Hwy., New Orleans, LA 70121. Tel: (504) 842-3980. Email: bbagert@ochsner.org

\section{INTRODUCTION}

Magnetic resonance imaging (MRI) enhanced with gadolinium-based contrast agents (GBCAs) is an essential component in the diagnosis and management of a wide variety of neurologic diseases, including demyelination, malignancy, and infections. ${ }^{1}$ The US Food and Drug Administration (FDA) approved GBCAs for clinical use in 1988, and they are generally regarded as having had an excellent safety profile for the past 30 years. However, several recent papers (1995-2018) have indicated that gadolinium has the potential to deposit and persist within the body, particularly within the dentate nucleus (DN) and globus pallidus (GP) of the brain. ${ }^{2-13}$ While the long-term clinical significance of gadolinium deposition remains unclear, this discovery has many in the medical community reconsidering the applications and necessity of GBCAs. In this article, we review the peer-reviewed data on GBCA safety and deposition. We also discuss the latest medical society recommendations and perspectives on GBCA use in clinical practice.

\section{BACKGROUND, METABOLISM, AND EXCRETION}

The first GBCA, gadopentetate, received FDA approval in 1988. ${ }^{14}$ Since that time, eight other GBCAs have received
FDA approval (Table 1)..$^{14-18}$ By 2016, more than 300 million patients had received GBCAs. ${ }^{19}$

GBCAs are used clinically in the diagnosis and management of many neurologic diseases. In the central nervous system, GBCAs are used in contrast-enhanced MRI, perfusion imaging, and magnetic resonance angiography. ${ }^{1}$ GBCAs are paramagnetic compounds that are imaged indirectly through their influence on the hydrogen relaxivity of surrounding tissue water molecules that is most readily visible on T1-weighted MRI sequences. ${ }^{20-22}$ This change in hydrogen relaxivity allows visualization of pathologies that may not otherwise be apparent and can significantly increase the sensitivity of the MRI. ${ }^{20}$ Increased T1 signal (or T1 shortening) after administration of a GBCA may reflect areas of neovascularity or disruption of the bloodbrain barrier, as GBCAs do not cross the blood-brain barrier in significant quantities. ${ }^{23,24}$ The pattern and distribution of this change in signal after GBCA administration, known as enhancement, can also increase the specificity of an MRI, as seen when MRI is used to differentiate between high- and low-grade gliomas. ${ }^{24}$ Contrast-enhanced MRI also improves the diagnostic sensitivity of MRI in numerous disease processes, including multiple sclerosis (MS), malignancy, and 
Table 1. US Food and Drug Administration-Approved Gadolinium-Based Contrast Agents ${ }^{\text {14-18 }}$

\begin{tabular}{|c|c|c|c|c|}
\hline Generic Name & Brand Name & Date Approved & Chemical Structure & Indication \\
\hline $\begin{array}{l}\text { Gadopentetate } \\
\text { dimeglumine }\end{array}$ & Magnevist & $6 / 2 / 1988$ & Linear & $\begin{array}{l}\text { Central nervous system, } \\
\text { extracranial/extraspinal, body }\end{array}$ \\
\hline Gadoteridol & ProHance & 11/16/1992 & Macrocyclic & $\begin{array}{l}\text { Central nervous system, } \\
\text { extracranial/extraspinal }\end{array}$ \\
\hline Gadoversetamide & OptiMARK & 12/8/1999 & Linear & Central nervous system, liver \\
\hline $\begin{array}{l}\text { Gadobenate } \\
\text { dimeglumine }\end{array}$ & MultiHance & $11 / 23 / 2004$ & Linear & $\begin{array}{l}\text { Central nervous system, renal or } \\
\text { aortoiliofemoral occlusive } \\
\text { vascular disease }\end{array}$ \\
\hline Gadodiamide & Omniscan & 9/5/2007 & Linear & Central nervous system, body \\
\hline Gadoxetate disodium & Eovist & $7 / 3 / 2008$ & Linear & Liver lesions \\
\hline Gadofosveset trisodium & $\begin{array}{l}\text { Ablavar (formerly } \\
\text { Vasovist) }\end{array}$ & $12 / 22 / 2008$ & Linear & Aortoiliac occlusive disease \\
\hline Gadobutrol & Gadavist & $3 / 14 / 2011$ & Macrocyclic & Central nervous system \\
\hline Gadoterate meglumine & Dotarem & $3 / 20 / 2013$ & Macrocyclic & Central nervous system \\
\hline
\end{tabular}

infections. ${ }^{1,25-28}$ In addition to use in the central nervous system, GBCAs are also used in numerous body, cardiac, and musculoskeletal imaging applications. ${ }^{1}$

Gadolinium is a rare earth element. ${ }^{16,20}$ GBCAs are composed of gadolinium with a chelating ligand in either linear ${ }^{29}$ or macrocyclic ${ }^{30}$ form (Figure 1) and are administered intravenously during a magnetic resonance examination. ${ }^{1,16,20,31}$ The chelating ligand limits potential toxicity from ionized gadolinium $\left(\mathrm{Gd}^{3+}\right)^{3,21}$ that may otherwise have interfered with calcium-binding receptors. ${ }^{32}$ Dissociation of chelated gadolinium occurs in the serum through a combination of dechelation and the ligand-metal (eg, zinc, copper, iron) exchange of transmetalation. ${ }^{31,32}$

The thermal and kinetic stabilities of each GBCA are thought to contribute to the differences in the rate of gadolinium deposition. ${ }^{31,32}$ Macrocyclic GBCAs are thought to offer more secure binding of $\mathrm{Gd}^{3+}$ as evidenced by studies in animals ${ }^{3,33}$ and in vitro studies in human serum ${ }^{31}$ in which increased gadolinium dissociation and retention generally correlated with lower chemical stability of linear GBCAs. The increased stability of macrocyclic GBCAs may be related to a more rigid cage-like structure of the ligand with which to bind $\mathrm{Gd}^{3+}$, leading to less dissociation of $\mathrm{Gd}^{3+}$ compared to linear GBCAs. ${ }^{16,31}$ Because GBCAs are primarily cleared by the kidney (notably, gadoxetate disodium is equally cleared by renal and hepatobiliary routes), decreased renal function leads to prolonged GBCA exposure, allowing increased time for gadolinium deposition. ${ }^{14,20,31}$

\section{EVIDENCE OF GADOLINIUM DEPOSITION IN TISSUE \\ Early Studies of Gadolinium Deposition}

In 1995, Tweedle et al demonstrated that radiolabeled gadolinium distributes into the liver and bones of mice and rats, providing initial evidence that gadolinium might persist in tissue. ${ }^{3}$ In 2006, White et al used inductively coupled plasma mass spectroscopy (ICP-MS) to demonstrate gadolinium deposition in human femurs 3-8 days after exposure to gadodiamide (linear GBCA; $n=9$ subjects) or gadoteridol (macrocyclic GBCA; $n=10$ subjects). ${ }^{34}$ The study showed that although both agents resulted in gadolinium deposition, the group exposed to linear GBCAs had higher levels of gadolinium deposition as measured by ICP-MS, suggesting a different rate of deposition between macrocyclic and linear GBCAs. ${ }^{34}$

\section{Studies of Gadolinium Deposition in the Animal Brain}

In August 2015, Robert et al reported results of a rat study in which they found that gadodiamide (linear GBCA) administration led to deposition in the deep cerebellar nuclei of rats as measured by MRI and correlated with ICP-MS. ${ }^{6}$ While the investigators did not detect gadolinium deposition on brain $\mathrm{MRI}$ in those exposed to the macrocyclic GBCA gadoterate, they did observe evidence of macrocyclic GBCA deposition in the brain when measured with ICP-MS. ${ }^{6}$

Two studies published in February 2016 examined the difference in gadolinium deposition between linear and macrocyclic GBCAs in healthy rats., ${ }^{9,10}$ Groups led by Jost et al and Robert et al examined rats exposed to linear and macrocyclic GBCAs. ${ }^{9,10}$ Both studies revealed increased signal on T1-weighted images on brain MRI in rats exposed to linear GBCAs but not in those exposed to macrocyclic GBCAs. ${ }^{9,10}$

\section{Studies of Gadolinium Deposition in the Human Brain}

The first report of suspected gadolinium deposition visualized on MRI in the human brain was a retrospective study published in 2014 by Kanda et al. ${ }^{5}$ The authors examined 19 patients who had undergone between 6 and 12 GBCA administrations and found $\mathrm{T} 1$ shortening on brain $\mathrm{MRI}$ in the DN and GP compared to controls. ${ }^{5}$ A study conducted by Errante et al examined patients with $M S$ ( $n=38$ subjects) and brain tumors ( $n=37$ subjects) with normal renal function exposed to the linear GBCA gadodiamide and found similar evidence of T1 shortening of the DN on brain MRI in patients with more than 6 gadodiamide administrations. ${ }^{35}$ Kanda et al published a follow-up study in June 2015 exploring the 


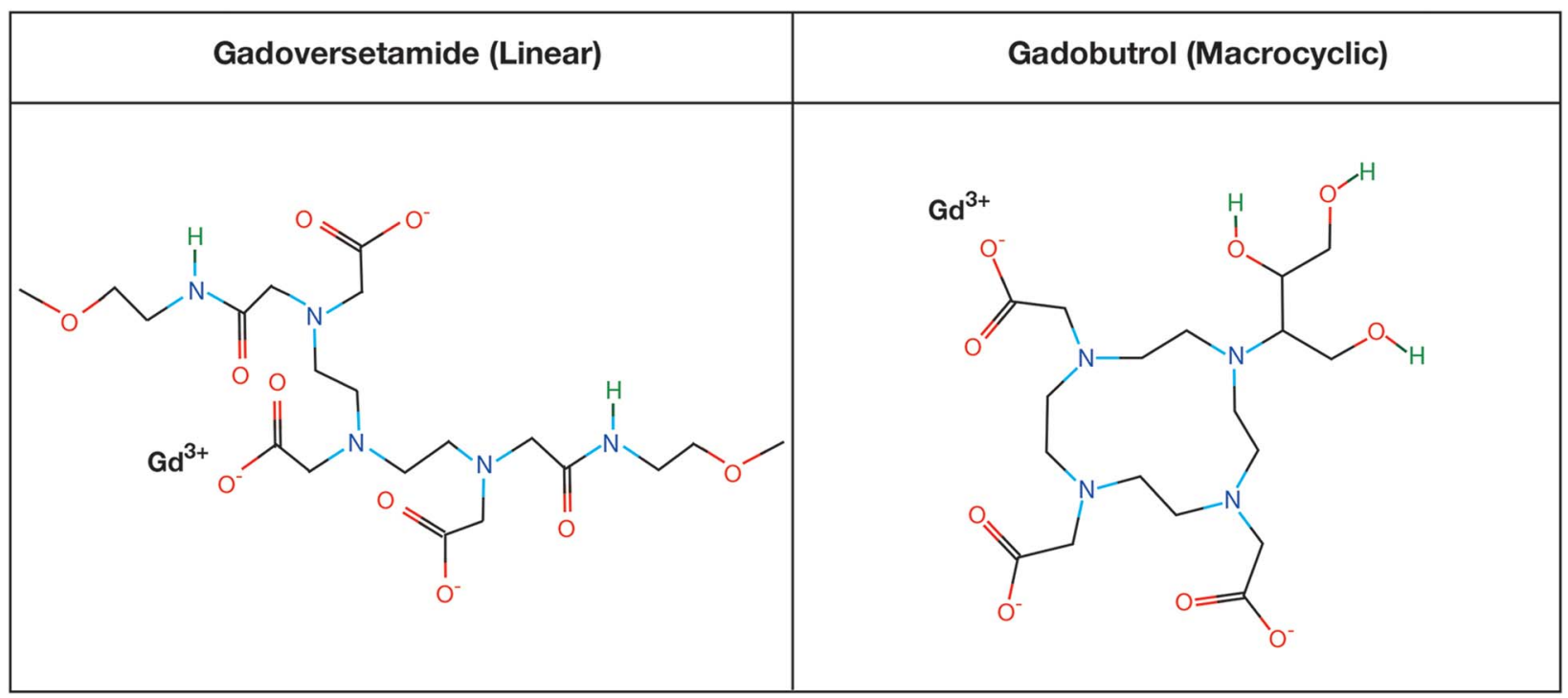

Figure 1. Chemical structures of example linear and macrocyclic and gadolinium-based contrast agents. ${ }^{29,30}$

difference between linear $(\mathrm{n}=23$ subjects) and macrocyclic ( $n=36$ subjects) GBCA gadolinium deposition in the brain. ${ }^{4}$ Using brain $\mathrm{MRI}$, the authors found a strong association between increased signal on T1-weighted images in the DN and linear GBCA exposure. ${ }^{4}$ This finding was not observed with macrocyclic GBCAs. ${ }^{4}$ In June 2015, Radbruch et al published a retrospective study examining subjects who had undergone at least 6 consecutive MRIs with either gadopentetate (linear; $n=50$ subjects) or gadoterate (macrocyclic; $\mathrm{n}=50$ subjects). ${ }^{36}$ Similar to Kanda et al, Radbruch et al reported a strong association between increased signal on T1-weighted images in those exposed to linear but not macrocyclic GBCAs. ${ }^{4,36}$ In July 2015, 1 month after the publication of these studies by Kanda et al and Radbruch et al, the FDA announced it would investigate the health impacts of gadolinium deposition in the brain. ${ }^{37}$

In March 2016, Stojanov et al published a study examining gadobutrol (macrocyclic) administration in 58 patients with relapsing-remitting MS and in contrast to prior studies, reported that the macrocyclic gadobutrol led to increased signal on T1-weighted images in the DN and GP on brain MRI. ${ }^{2}$ This study has been highly critiqued, with others expressing concerns about the validity of the results as noted in letters published in May $2016^{38}$ and December $2015 .{ }^{39}$ One of the primary drivers behind these concerns is the failure to account for prior GBCA exposures in this study. ${ }^{2}$ A reproducibility study conducted by Radbruch et al was published in 2015 in which the same macrocyclic GBCA, gadobutrol, was studied in 30 patients with brain tumors. ${ }^{40}$ In contrast to the study by Stojanov et al, Radbruch et al did not find evidence of signal increase in the DN and GP on T1-weighted brain MRI. 2,40 In contrast to Stojanov et al, Radbruch et al excluded all patients with more than 2 prior GBCA exposures. ${ }^{2,40}$

Quattrocchi et al published a retrospective study in 2015 examining 102 patients with meningiomas exposed to the linear agent gadodiamide that revealed increased signal on T1-weighted images in the DN after 6 or more contrast- enhanced MRls, suggesting a dose response. ${ }^{12}$ In this study, there were no reports of any significant neurologic complaints that the authors could associate with T1 shortening in the DN. ${ }^{12}$

From 2015 through 2016, 3 autopsy studies were conducted to assess for gadolinium deposition. ${ }^{7,8,41}$ In 2015, McDonald et al examined autopsies of 13 patients with normal renal function exposed to 4-29 administrations of the linear GBCA gadodiamide. ${ }^{7}$ At autopsy, tissue was examined for radiologic, spectrometric, electron microscopic, and histopathologic evidence of gadolinium deposition. ${ }^{7}$ The authors found evidence of dose-dependent signal changes on brain $\mathrm{MRI}$, as well as evidence of gadolinium deposition on ICP-MS and electron microscopy compared to controls. ${ }^{7}$ One month after the McDonald study was published, Kanda et al published an autopsy study in which 5 subjects with normal renal function who had been exposed to linear GBCAs (gadopentetate and gadodiamide) were found to have gadolinium deposition through ICP-MS in the DN and GP. ${ }^{41}$ In 2016, a study by Murata et al examined autopsies of 9 patients with normal renal function who had been exposed to GBCAs and found that all had demonstrated some degree of gadolinium deposition in the brain when measured with ICP-MS. In this cohort, 7 individuals had only been exposed to the macrocyclic GBCAs gadobutrol and gadoteridol. ${ }^{8}$

In 2018, Moser et al published a study of 119 patients with brain tumors who had been exposed to various forms of GBCAs and found that exposure to linear GBCAs led to increased signal on T1-weighted images, whereas similar changes were not observed with macrocyclic GBCAs. ${ }^{13}$ Vergauwen et al conducted a retrospective study in a population of patients with either von Hippel-Lindau disease $(n=28$ subjects) or tuberous sclerosis complex ( $n=24$ subjects) and discovered that both demonstrated increased signal on T1weighted images in the DN and GP, although the increase in the tuberous sclerosis complex group did not reach statistical significance. ${ }^{11}$ Notably, detailed records of the identity of contrast agents used for this study were not available, 

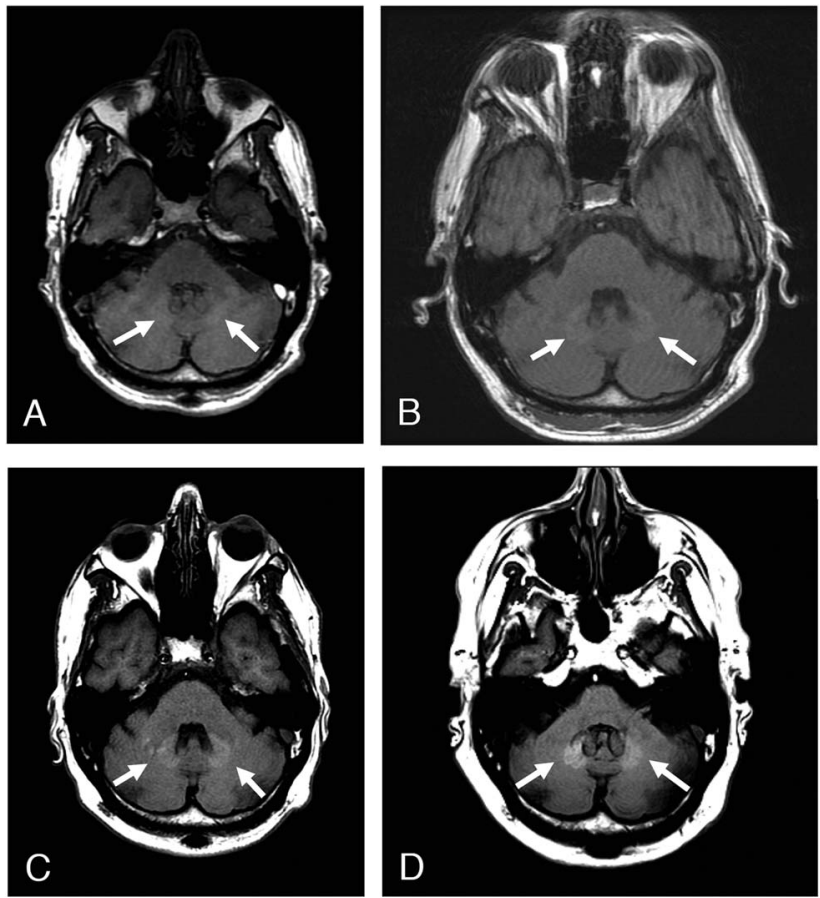

Figure 2. Sequential axial T1-weighted noncontrast magnetic resonance images through the posterior fossa of a 52-year-old male with history of multiple sclerosis demonstrate a progressive increase in signal within the dentate nuclei following repeated doses of gadolinium-based contrast agents in (A) December 2011, (B) June 2013, (C) September 2016, and (D) June 2018.

but basing a conjecture on the agents used by the hospitals where the MRIs were performed, linear GBCAs (gadopentetate, gadodiamide, gadobenate) were likely predominately used, with the recent introduction of gadoterate in 2016 (2 years prior to publication). ${ }^{11}$

In May 2017, the FDA announced that to date no harmful sequelae of gadolinium deposition in the brain had been identified but that reviews would continue. ${ }^{42}$ In May 2018, the FDA announced that GBCAs would require new class warnings that detail the potential for gadolinium deposition. ${ }^{18}$

\section{Summary of Evidence for Gadolinium Deposition in the Brain}

Evidence shows that gadolinium is deposited in the DN and GP of the brain, as measured by autopsy, spectrometry, and MRI. ${ }^{4-12,35,36,40,41}$ An example of the accumulation of gadolinium deposition is shown in Figure 2. Gadolinium deposition has been more prominently associated with linear than with macrocyclic GBCAs, ${ }^{4,6,8-10,13,36,40}$ possibly because of differences in kinetic and thermal stabilities. ${ }^{31,32}$ Additionally, evidence suggests a dose-dependent effect of gadolinium deposition. ${ }^{7,12,35}$ Although studies have revealed mixed evidence of gadolinium deposition with macrocyclic GBCAs on MRI, ${ }^{2,4,6,9,10,13,36,40}$ spectroscopic evidence suggests that macrocyclic GBCAs can result in gadolinium deposition. ${ }^{6,8}$ One hypothesis to explain these findings is that the rate of macrocyclic GBCA gadolinium deposition is too low for detection via MRI but detectable by ICP-MS. Prior publications have hypothesized that the GP may be uniquely susceptible to metal deposition as seen in other metabolic disorders, such as hepatic dysfunction, Fahr disease, and Wilson disease. ${ }^{5,7}$

\section{OTHER SAFETY CONSIDERATIONS}

Since their introduction in $1988,{ }^{14}$ GBCAs have garnered a good safety record. In 2018, Behzadi et al conducted a metaanalysis to examine adverse reactions to GBCAs and discovered that the rate of immediate allergic-like reactions to GBCAs was 9.2 per 10,000 administrations and of severe allergic-like reactions was 0.52 per 10,000 administrations. ${ }^{43}$ Furthermore, this analysis noted that linear nonionic GBCAs, such as gadodiamide in this metaanalysis, conferred the lowest risk of acute allergic reactions. ${ }^{43}$ In terms of acute adverse reactions, GBCAs compared favorably with intravenous (IV) iodinated contrast for computed tomography scans. Common adverse reactions to iodinated contrast media include allergic-like reactions and cutaneous reactions (such as urticaria and/or rash). ${ }^{19}$ The American College of Radiology (ACR) Manual on Contrast Media details overall adverse reaction rates ranging from $0.2 \%-0.7 \%$ depending on the study, while severe reactions to IV iodinated contrast have been reported at a rate of 4 per 10,000. ${ }^{19}$

In 2000, the first case series of nephrogenic systemic fibrosis (NSF), a scleroderma-like illness initially labeled nephrogenic fibrosing dermopathy, was published. ${ }^{44,45}$ NSF was found to be associated with GBCA exposure in patients with decreased renal function (acute renal failure, chronic renal failure with glomerular filtration rate $<30 \mathrm{~mL} / \mathrm{min} / \mathrm{m}^{2}$ ). ${ }^{44-46}$ The ACR classifies GBCAs based on their association with reported cases of NSF. The group I agents, consisting of gadodiamide, gadopentetate dimeglumine, and gadoversetamide, are all linear agents and associated with the greatest number of NSF cases. ${ }^{47}$ The incidence of NSF has dramatically decreased in the past decade by limiting GBCA use in high-risk populations, using more stable GBCAs, and decreasing the dose of GBCAs for patients with poor kidney function. ${ }^{46}$

Ray et al published a population-based cohort study examining the association between MRI exposure during pregnancy and adverse fetal and childhood outcomes. ${ }^{48}$ The authors found an increased risk of rheumatologic, inflammatory, infiltrative skin conditions and stillbirth or neonatal death in patients exposed to GBCAs during pregnancy. ${ }^{48}$ They did not find an increased risk of conditions resembling NSF in the children exposed to GBCAs in utero. ${ }^{48}$ At this time, GBCA use in pregnancy is typically contraindicated.

Despite the evidence of gadolinium deposition in the brain, evidence for adverse health effects resulting from gadolinium deposition is limited. In 2016, Semelka et al published an initial description of symptomatology following gadolinium exposure they termed "gadolinium deposition disease." 49 For this report, Semelka et al posted an online survey consisting of 18 questions to a private blog (MRI-GadoliniumToxicity Support Group) and a public MRI Gadolinium Toxicity Facebook page to identify symptoms of gadolinium deposition. ${ }^{49}$ Inclusion criteria required self-reported normal renal function, onset of symptoms ranging from 1-365 days after gadolinium exposure, and self-reported laboratory evidence of gadolinium presence in the respondent's body. ${ }^{49}$ 
Table 2. Society Recommendations Regarding Gadolinium-Based Contrast Agent Use ${ }^{16,19,51,52}$

\begin{tabular}{|c|c|c|}
\hline Society & Summary of Statements and Recommendations & Date \\
\hline \multirow[t]{5}{*}{$\begin{array}{l}\text { National Institutes of } \\
\text { Health }^{16}\end{array}$} & $\begin{array}{l}\text { 1. GBCAs should only be used when clinically indicated or when specified in an } \\
\text { institutional review board-approved protocol. }\end{array}$ & March 2016 \\
\hline & $\begin{array}{l}\text { 2. When GBCAs are required, consider the use of a macrocyclic GBCA (eg, gadobutrol, } \\
\text { gadoteridol, or gadoterate meglumine) rather than a linear agent. }\end{array}$ & \\
\hline & $\begin{array}{l}\text { 3. For patients with documented sensitivity (eg, hives) to macrocyclic agents, it is } \\
\text { appropriate to use linear agents when clinically indicated. }\end{array}$ & \\
\hline & $\begin{array}{l}\text { 4. MRI protocols should always consider FDA label indications and dosing schemes for } \\
\text { administration of GBCAs. }\end{array}$ & \\
\hline & $\begin{array}{l}\text { 5. Encourage intradepartmental and interdepartmental research programs to evaluate } \\
\text { T1 shortening in the brain and other organs in patients who have received multiple } \\
\text { doses of GBCAs. }\end{array}$ & \\
\hline $\begin{array}{l}\text { American College of } \\
\text { Radiology }\end{array}$ & $\begin{array}{l}\text { Would be prudent to consider the clinical benefit of the diagnostic information or } \\
\text { treatment result that MRI or MRA may provide against the unknown potential risk of } \\
\text { gadolinium deposition in the brain for each individual patient. Particular attention } \\
\text { should be paid to pediatric and other patients who may receive many } \\
\text { GBCA-enhanced MRI studies over the course of their lifetimes. If the decision for an } \\
\text { individual patient is made to use a GBCA for an MRI study, multiple factors need to } \\
\text { be considered when selecting a GBCA, including diagnostic efficacy, relaxivity, rate } \\
\text { of adverse reactions, dosing/concentration, and propensity to deposit in more } \\
\text { sensitive organs such as the brain. As this gadolinium deposition phenomenon } \\
\text { remains a relatively undefined clinical phenomenon, and accurate and complete } \\
\text { data may be useful as investigations proceed, the identity and dose of GBCA used } \\
\text { should be recorded after each intravenous administration. }\end{array}$ & May 2016 \\
\hline \multirow[t]{4}{*}{$\begin{array}{l}\text { International Society for } \\
\text { Magnetic Resonance in } \\
\text { Medicine }^{52}\end{array}$} & $\begin{array}{l}\text { 1. The ISMRM urges caution in the use of any medical compound, including GBCAs. Per } \\
\text { standard practice, use of GBCAs should be avoided when not necessary. The } \\
\text { evidence on gadolinium deposition emphasizes but does not alter this practice, and } \\
\text { GBCAs should not be withheld from patients with a clinical indication for } \\
\text { gadolinium-enhanced MRI. The physician responsible for the administration of a } \\
\text { contrast agent should understand the benefits and risks of the agent. }\end{array}$ & July 2017 \\
\hline & $\begin{array}{l}\text { 2. The clinical indication for which a GBCA is administered, the specific contrast agent } \\
\text { used, its dosage, and other pertinent information should be documented in the } \\
\text { patient's medical record. }\end{array}$ & \\
\hline & $\begin{array}{l}\text { 3. Some commercially available macrocyclic agents might deposit less gadolinium } \\
\text { than some linear agents; however, evidence shows that gadolinium deposition in } \\
\text { the brain can also occur after the administration of macrocyclic agents. Evidence } \\
\text { suggests differences in gadolinium deposition rates among macrocyclic agents and } \\
\text { among linear agents, although some data are discordant. Relaxivity differences } \\
\text { between contrast agents and between the potentially deposited chemical species } \\
\text { can complicate the interpretation of differences in signal intensity. No evidence } \\
\text { shows any harmful effects from the deposition of gadolinium, and therefore } \\
\text { whether use of macrocyclic agents should be favored over linear agents is unclear. } \\
\text { When choosing a contrast agent, many factors should be considered, including } \\
\text { pharmacokinetics, relaxivity, efficacy, potential side effects (such as allergic } \\
\text { reactions), patient age, probability of the need for repeated examinations, and cost. } \\
\text { Institutions should weigh these factors and consider that some agents might have a } \\
\text { greater propensity for deposition than others. }\end{array}$ & \\
\hline & $\begin{array}{l}\text { 4. Given the importance of GBCAs for advancing scientific discovery and improving } \\
\text { clinical care, the ISMRM Safety Committee supports the views of the National } \\
\text { Institutes of Health, in that administration of GBCAs is appropriate in research } \\
\text { settings under the guidance of protocols approved by an institutional review board, } \\
\text { and that must include patient's informed consent. Because no risks are known to be } \\
\text { associated with gadolinium deposition in the brain, the ISMRM is unable to make an } \\
\text { overarching recommendation regarding disclosure of gadolinium deposition to } \\
\text { research participants. Therefore, each institution must decide whether inclusion of } \\
\text { information on the deposition of gadolinium in the brain is necessary and should be }\end{array}$ & \\
\hline
\end{tabular}


Table 2. Continued

\begin{tabular}{|c|c|c|}
\hline Society & Summary of Statements and Recommendations & Date \\
\hline & $\begin{array}{l}\text { included as part of the consent form; if so, the institution must decide on the } \\
\text { description to use. The circumstances under which the GBCA is administered, the } \\
\text { unknown risks of gadolinium deposition, and the need to explain the deposition to } \\
\text { participants in appropriate language should be taken into account. In the event that } \\
\text { new data are available describing adverse biological or clinical effects associated } \\
\text { with gadolinium deposition subsequent to this Personal View, it might be } \\
\text { appropriate to include that information as part of the consent process. }\end{array}$ & \\
\hline & $\begin{array}{l}\text { 5. Investigators reporting studies on gadolinium deposition in the brain should } \\
\text { exercise meticulous disclosure of financial, consulting, and advising relationships } \\
\text { with industries as potential conflicts of interest. Although proper disclosure of } \\
\text { conflicts of interest must be done for all academic publications, transparency is } \\
\text { particularly relevant for studies of gadolinium deposition. }\end{array}$ & \\
\hline & $\begin{array}{l}\text { 6. Because of the potential confounding of disease-related signal intensity changes } \\
\text { with gadolinium deposition, future studies should explicitly describe all relevant } \\
\text { clinical history of participants, including treatment of the patients in the study. }\end{array}$ & \\
\hline \multirow[t]{7}{*}{$\begin{array}{l}\text { Consortium of Multiple } \\
\text { Sclerosis Centers }\end{array}$} & $\begin{array}{l}\text { GBCAs do accumulate in the brain and to a much lesser degree with macrocyclic } \\
\text { agents. While no known CNS toxicity has been identified with the use of GBCAs, } \\
\text { these agents should be used judiciously, recognizing that gadolinium continues to } \\
\text { play an invaluable role in specific circumstances related to the diagnosis and } \\
\text { follow-up of individuals with MS. }\end{array}$ & $\begin{array}{l}\text { February } \\
2018\end{array}$ \\
\hline & MS GBCA use is essential for & \\
\hline & - following a patient with highly active disease & \\
\hline & $\begin{array}{l}\text { - when there is rapidly declining and unexplained and unexpected clinical worsening } \\
\text { occurs }\end{array}$ & \\
\hline & - when there is concern regarding an alternative diagnosis other than MS & \\
\hline & MS GBCA use is optional for & \\
\hline & $\begin{array}{l}\text { the follow-up monitoring of patients with MS to detect subclinical disease activity } \\
\text { that could lead to a change in therapy; the use of GBCA may be helpful within the } \\
\text { first } 2 \text { years of treatment onset but is not required because new T2 MS lesions can be } \\
\text { identified on well-performed MRI using a standardized protocol unless there is a } \\
\text { large T2 lesion burden. }\end{array}$ & \\
\hline
\end{tabular}

CNS, central nervous system; FDA, US Food and Drug Administration; GBCA, gadolinium-based contrast agent; ISMRM, International Society for Magnetic Resonance in Medicine; MRA, magnetic resonance angiography; MRI, magnetic resonance imaging; MS, multiple sclerosis.

Forty-two individuals participated in the survey. Symptoms reported included pain, skin thickening and discoloration, joint stiffness, fatigue, persistent clouded mentation, dry eyes, and vision problems. ${ }^{49}$

In 2016, Burke et al published the results of a similar survey. This study (Semelka was a coauthor) also searched for adverse symptoms of gadolinium deposition based on survey questions posted to the private MRIGadolinium-Toxicity Support Group and the public MRI Gadolinium Toxicity Facebook page. ${ }^{50}$ Fifty individuals participated in the survey, of whom a majority reported joint pain, headaches, flu-like symptoms, skin changes, digestive symptoms, difficulty breathing, and generalized whole-body symptoms. ${ }^{50}$

Both studies have several striking scientific limitations. Chief among them is the selection bias in targeting this survey to a study population comprised exclusively of individuals who a priori believe they have symptoms related to gadolinium exposure. ${ }^{49,50}$ This selection bias undermines the results and calls into question the validity of any conclusions drawn by the authors. As a retrospective study, these studies were prone to bias and confounding even beyond the selection bias described. While Semelka et al included inclusion criteria, it is not clear that the participants were verified to have met these criteria. ${ }^{49}$ Neither study included any exclusion criteria or a control group. ${ }^{49,50}$ Furthermore, both surveys were conducted online, raising questions regarding the authenticity of the participants. ${ }^{49,50}$ Semelka et al report that 3 random individuals were examined by a physician with experience in NSF, but no objective results of the examination are detailed. ${ }^{49}$ Neither study reports the original indications for MRI, although the authors indicate that the symptoms reported in the survey are new since the respondents underwent contrast-enhanced MRI. ${ }^{49,50}$

Overall, while these studies have attempted to answer the ongoing question regarding the clinical significance of gadolinium deposition, ${ }^{49,50}$ they fall short of the scientific standards of clinical investigation. We did not find a peerreviewed report with sufficient scientific rigor to associate gadolinium deposition with adverse effects in humans. 


\section{SOCIETY RECOMMENDATIONS AND FUTURE DIRECTIONS}

The ACR, ${ }^{19}$ Consortium of Multiple Sclerosis Centers (CMSC), ${ }^{51}$ International Society for Magnetic Resonance in Medicine (ISMRM), ${ }^{52}$ and National Institutes of Health (NIH) ${ }^{16}$ have made recommendations regarding GBCA use given recent evidence of gadolinium deposition (Table 2).

In January 2017, the CMSC released updated MRI guidelines for providers addressing GBCA accumulation in the brain and recommending judicious use given the invaluable role GBCAs can play in the diagnosis and management of individuals with MS. ${ }^{53}$ The 2018 CMSC guidelines included more specific recommendations and distinguished between "essential" and "optional" uses of GBCAs. Essential uses include MRI surveillance in patients with highly active disease or rapid clinical worsening or for patients with diagnostic uncertainty. ${ }^{51}$ Optional uses of GBCAs include follow-up monitoring of MS patients to detect subclinical disease activity. ${ }^{51}$ Additionally, the CMSC notes that a well-performed noncontrast MRI may be used to detect new T2 lesions in patients without a high lesion burden. ${ }^{51}$

Similarly, the ACR published a position statement regarding gadolinium deposition in 2016 that was maintained in the 2017 Manual on Contrast Media. ${ }^{19}$ This statement acknowledged that some GBCAs may be more likely to cause gadolinium deposition but that the potential adverse effects of this deposition, if any, remain to be elucidated and recommended that further research be done. ${ }^{19,47}$ The ACR recommended weighing the clinical benefit of GBCA use with the unknown potential risk of gadolinium deposition ${ }^{19,47}$ and documenting all GBCA administration in the patient's medical record, including the GBCA identity, dose, and date of administration. ${ }^{19}$

In 2016, the NIH published GBCA guidelines as well, noting that linear GBCAs may continue to be used in a limited fashion in individuals who are allergic to macrocyclic agents or in specific indications such as liver imaging. ${ }^{16}$

In 2017, the ISMRM published guidelines that echoed many of the above statements. ${ }^{52}$ The ISMRM reinforced that the evidence for gadolinium deposition to date is not an indication to withhold GBCAs in a situation in which they are otherwise clinically indicated. ${ }^{52}$

Efforts are underway to develop alternative contrast agents to GBCAs, with manganese-based and ironbased complexes undergoing investigation. ${ }^{54-56}$ Additionally, the use of different noncontrast magnetic resonance sequences as substitutes for GBCA administration are being explored. ${ }^{57,58}$ In the future, these modalities may become viable alternatives to GBCA use.

\section{CONCLUSION}

Strong evidence shows that repeated GBCA exposure can lead to gadolinium deposition in the DN and GP in the brain. Evidence to date suggests that macrocyclic GBCAs are far less likely than linear agents to cause gadolinium deposition in brain tissue visible on T1-weighted MRI. Given this evidence, multiple medical societies have recommended preferential use of macrocyclic GBCAs instead of linear GBCAs. Despite the evidence of gadolinium deposition, no convincing data so far have linked gadolinium deposition with adverse health outcomes. Further investigation with scientific rigor is needed to explore this question.

Since 1988, GBCAs have been safely administered to millions of patients globally, and their use has undoubtedly benefited innumerable patients by enabling clinicians to make earlier and more accurate diagnoses of neurologic disease. Considering the benefits and unknowns of GBCAs, providers should use these agents judiciously and engage in shared decision-making with patients, with the discussion including an individualized risk-benefit assessment.

\section{ACKNOWLEDGMENTS}

The authors have no financial or proprietary interest in the subject matter of this article.

\section{REFERENCES}

1. Lohrke J, Frenzel T, Endrikat J, et al. 25 years of contrast-enhanced MRI: developments, current challenges and future perspectives. Adv Ther. 2016 Jan;33(1):1-28. doi: 10.1007/s12325-015-0275-4.

2. Stojanov DA, Aracki-Trenkic A, Vojinovic S, Benedeto-Stojanov $D$, Ljubisavljevic S. Increasing signal intensity within the dentate nucleus and globus pallidus on unenhanced T1W magnetic resonance images in patients with relapsing-remitting multiple sclerosis: correlation with cumulative dose of a macrocyclic gadolinium-based contrast agent, gadobutrol. Eur Radiol. 2016 Mar;26(3):807-815. doi: 10.1007/s00330-015-3879-9.

3. Tweedle MF, Wedeking P, Kumar K. Biodistribution of radiolabeled, formulated gadopentetate, gadoteridol, gadoterate, and gadodiamide in mice and rats. Invest Radiol. 1995;30(6):372-380.

4. Kanda $T$, Osawa $M, O b a ~ H$, et al. High signal intensity in dentate nucleus on unenhanced T1-weighted MR images: association with linear versus macrocyclic gadolinium chelate administration. Radiology. 2015 Jun;275(3):803-809. doi: 10.1148/radiol.14140364.

5. Kanda T, Ishii K, Kawaguchi H, Kitajima K, Takenaka D. High signal intensity in the dentate nucleus and globus pallidus on unenhanced T1-weighted MR images: relationship with increasing cumulative dose of a gadolinium-based contrast material. Radiology. 2014 Mar;270(3):834-841. doi: 10.1148/radiol.13131669.

6. Robert P, Lehericy S, Grand S, et al. T1-weighted hypersignal in the deep cerebellar nuclei after repeated administrations of gadolinium-based contrast agents in healthy rats: difference between linear and macrocyclic agents. Invest Radiol. 2015 Aug;50(8):473-480. doi: 10.1097/RLI.0000000000000181.

7. McDonald RJ, McDonald JS, Kallmes DF, et al. Intracranial gadolinium deposition after contrast-enhanced MR imaging. Radiology. 2015 Jun;275(3):772-782. doi: 10.1148/radiol.15150025.

8. Murata N, Gonzalez-Cuyar LF, Murata K, et al. Macrocyclic and other non-group 1 gadolinium contrast agents deposit low levels of gadolinium in brain and bone tissue: preliminary results from 9 patients with normal renal function. Invest Radiol. 2016 Jul;51(7):447-453. doi: 10.1097/RLI.0000000000000252.

9. Jost G, Lenhard DC, Sieber MA, Lohrke J, Frenzel T, Pietsch H. Signal increase on unenhanced $\mathrm{T} 1$-weighted images in the rat brain after repeated, extended doses of gadolinium-based contrast agents: comparison of linear and macrocyclic agents. Invest Radiol. 2016 Feb;51(2):83-89.

doi: $10.1097 /$ RLI.0000000000000242. 
10. Robert $P$, Violas $X$, Grand $S$, et al. Linear gadolinium-based contrast agents are associated with brain gadolinium retention in healthy rats. Invest Radiol. 2016 Feb;51(2):73-82. doi: 10.1097/RLI.0000000000000241.

11. Vergauwen E, Vanbinst AM, Brussaard C, et al. Central nervous system gadolinium accumulation in patients undergoing periodical contrast MRI screening for hereditary tumor syndromes. Hered Cancer Clin Pract. 2018 Jan 5;16:2. doi: 10.1186/s13053-017-0084-7.

12. Quattrocchi CC, Mallio CA, Errante Y, et al. Gadodiamide and dentate nucleus $\mathrm{T} 1$ hyperintensity in patients with meningioma evaluated by multiple follow-up contrast-enhanced magnetic resonance examinations with no systemic interval therapy. Invest Radiol. 2015 Jul;50(7):470-472. doi: 10.1097/RLI.0000000000000154.

13. Moser FG, Watterson CT, Weiss $S$, et al. High signal intensity in the dentate nucleus and globus pallidus on unenhanced T1-weighted mr images: comparison between gadobutrol and linear gadolinium-based contrast agents. AJNR Am J Neuroradiol. 2018 Feb 1. doi: 10.3174/ajnr.A5538.

14. Drugs@FDA: FDA approved drug products. U.S. Food and Drug Administration. www.accessdata.fda.gov/scripts/cder/daf/ index.cfm. Published 2018. Accessed June 14, 2018.

15. Aime S, Caravan P. Biodistribution of gadolinium-based contrast agents, including gadolinium deposition. J Magn Reson Imaging. 2009 Dec;30(6):1259-1267. doi: 10.1002/jmri.21969.

16. Malayeria AA, Brooks KM, Bryant LH, et al. National Institutes of Health perspective on reports of gadolinium deposition in the brain. J Am Coll Radiol. 2016 Mar;13(3):237-241. doi: 10.1016/j.jacr.2015.11.009.

17. Pinter NK, Klein JP, Mechtler LL. Potential safety issues related to the use of gadolinium-based contrast agents. Continuum (Minneap Minn). 2016 Oct;22(5, Neuroimaging):1678-1684. doi: $10.1212 / C O N .0000000000000378$.

18. FDA Drug Safety Communication: FDA warns that gadolinium-based contrast agents (GBCAs) are retained in the body; requires new class warnings [press release]. U.S. Food and Drug Administration. www.fda.gov/Drugs/DrugSafety/ ucm589213.htm. Updated May 16, 2018. Accessed January 18, 2019.

19. Manual on Contrast Media. v.10.3. Reston, VA: American College of Radiology; 2017.

20. Weinmann HJ, Brasch RC, Press WR, Wesbey GE. Characteristics of gadolinium-DTPA complex: a potential $\mathrm{nmr}$ contrast agent. AJR Am J Roentgenol. 1984;142:619-624. doi: 10.2214/ajr.142.3.619.

21. Lauffer RB. Paramagnetic metal complexes as water proton relaxation agents for NMR imaging: theory and design. Chem Rev. 1987 Oct;87(5):901-927. doi: 10.1021/cr00081a003.

22. Caravan P, Ellison JJ, McMurry TT, Lauffer RB. Gadolinium(III) chelates as MRI contrast agents: structure, dynamics, and applications. Chem Rev. 1999 Sep 8;99(9):2293-2352.

23. Aksoy D, Bammer R, Mlynash M, et al. Magnetic resonance imaging profile in blood-brain barrier injury in patients with acute intracerebral hemorrhage. J Am Heart Assoc. 2013 May 24;2(3):e000161. doi: 10.1161/JAHA.113.000161.

24. Gao H, Jiang X. Progress on the diagnosis and evaluation of brain tumors. Cancer Imaging. 2013 Dec 11;13(4):466-481. doi: 10.1102/1470-7330.2013.0039.

25. Hjort N, Wu O, Ashkanian M, et al. MRI detection of early blood-brain barrier disruption: parenchymal enhancement predicts focal hemorrhagic transformation after thrombolysis. Stroke. 2008 Mar;39(3):1025-1028. doi: 10.1161/STROKEAHA.107.497719.
26. Montagne A, Toga AW, Zlokovic BV. Blood-brain barrier permeability and gadolinium: benefits and potential pitfalls in research. JAMA Neurol. 2016 Jan;73(1):13-14. doi: 10.1001/jamaneurol.2015.2960.

27. Bastianello S, Pozzilli C, Bernardi S, et al. Serial study of gadolinium-DTPA MRI enhancement in multiple sclerosis. Neurology. 1990 Apr;40(4):591-595.

28. Stone LA, Smith ME, Albert PS, et al. Blood-brain barrier disruption on contrast-enhanced MRI in patients with mild relapsing-remitting multiple sclerosis: relationship to course, gender, and age. Neurology. 1995 Jun;45(6):1122-1126.

29. Gadoversetamide. Compound summary for CID 444013. National Center for Biotechnology Information. pubchem.ncbi.nlm.nih.gov/compound/444013. Published June 24, 2005. Accessed July 14, 2018.

30. Gadobutrol. Compound summary for CID 15814656. National Center for Biotechnology Information.pubchem.ncbi.nIm.nih. gov/compound/15814656. Published February 12, 2007. Accessed July 14, 2018.

31. Frenzel T, Lengsfeld $P$, Shirmer $H$, Hütter J, Weinmann HJ. Stability of gadolinium-based magnetic resonance imaging contrast agents in human serum at 37 degrees $C$. Invest Radiol. 2008 Dec;43(12):817-828. doi: 10.1097/RLI.0b013e3181852171.

32. Port M, Idée JM, Medina C, Robic C, Sabatou M, Corot C. Efficiency, thermodynamic and kinetic stability of marketed gadolinium chelates and their possible clinical consequences: a clinical review. Biometals. 2008 Aug;21(4):469-490. doi: 10.1007/s10534-008-9135-x.

33. Wedeking P, Kumar K, Tweedle MF. Dissociation of gadolinium chelates in mice: relationship to chemical characteristics. Magn Reson Imaging. 1992;10(4):641-648.

34. White GW, Gibby WA, Tweedle MF. Comparison of Gd(DTPA-BMA) (Omniscan) versus Gd(HP-DO3A) (ProHance) relative to gadolinium retention in human bone tissue by inductively coupled plasma mass spectroscopy. Invest Radiol. 2006 Mar;41(3):272-278. doi: 10.1097/01.rli.0000186569.32408.95.

35. Errante Y, Cirimele V, Mallio CA, Lazzaro VD, Zobel BB, Quattrocchi CC. Progressive increase of T1 signal intensity of the dentate nucleus on unenhanced magnetic resonance images is associated with cumulative doses of intravenously administered gadodiamide in patients with normal renal function, suggesting dechelation. Invest Radiol. 2014 Oct;49(10):685-690. doi: 10.1097/RLI.0000000000000072.

36. Radbruch A, Weberling LD, Kieslich PJ, et al. Gadolinium retention in the dentate nucleus and globus pallidus is dependent on the class of contrast agent. Radiology. 2015 Jun;275(3):783-791. doi: 10.1148/radiol.2015150337.

37. FDA Drug Safety Communication: FDA evaluating the risk of brain deposits with repeated use of gadolinium-based contrast agents for magnetic resonance imaging (MRI). U.S. Food and Drug Administration. www.fda.gov/Drugs/DrugSafety/ ucm455386.htm. Published July 27, 2015. Updated May 22, 2017. Accessed January 18, 2019.

38. Kanda T, Oba H, Toyoda K, Furui S. Macrocyclic gadolinium-based contrast agents do not cause hyperintensity in the dentate nucleus. AJNR Am J Neuroradiol. 2016 May;37(5):E41. doi: 10.3174/ajnr.A4710.

39. Runge VM. Macrocyclic versus linear gadolinium chelates. Invest Radiol. 2015 Dec;50(12):811. doi: 10.1097/RLI.0000000000000229.

40. Radbruch A, Weberling LD, Kieslich PJ, et al. High-signal intensity in the dentate nucleus and globus pallidus on unenhanced T1-weighted images: evaluation of the 
macrocyclic gadolinium-based contrast agent gadobutrol. Invest Radiol. 2015 Dec;50(12):805-810. doi: 10.1097/RLI.0000000000000227.

41. Kanda T, Fukusato T, Matsuda M, et al. Gadolinium-based contrast agent accumulates in the brain even in subjects without severe renal dysfunction: evaluation of autopsy brain specimens with inductively coupled plasma mass spectroscopy. Radiology. 2015 Jul;276(1):228-232. doi: 10.1148/radiol.2015142690.

42. FDA Drug Safety Communication: FDA identifies no harmful effects to date with brain retention of gadolinium-based contrast agents for MRIs; review to continue [press release]. U.S. Food and Drug Administration. www.fda.gov/Drugs/ DrugSafety/ucm559007.htm. Published May 22, 2017. Updated December 19, 2017. Accessed January 18, 2019.

43. Behzadi AH, Zhao Y, Farooq Z, Prince MR. Immediate allergic reactions to gadolinium-based contrast agents: a systematic review and meta-analysis. Radiology. 2018 Feb;286(2):471-482. doi: 10.1148/radiol.2017162740.

44. Cowper SE, Robin HS, Steinberg SM, Su LD, Gupta S, LeBoit PE. Scleromyxoedema-like cutaneous diseases in renal-dialysis patients. Lancet. 2000 Sept 16;356(9234):1000-1001. doi: 10.1016/S0140-6736(00)02694-5.

45. Cowper SE, Bucala R, Leboit PE. Nephrogenic fibrosing dermopathy/nephrogenic systemic fibrosis - setting the record straight. Semin Arthritis Rheum. 2006 Feb;35(4): 208-210.

46. Altun E, Martin DR, Wertman R, Lugo-Somolinos A, Fuller ER 3rd, Semelka RC. Nephrogenic systemic fibrosis: change in incidence following a switch in gadolinium agents and adoption of a gadolinium policy-report from two U.S. universities. Radiology. 2009 Dec;253(3):689-696. doi: $10.1148 /$ radiol.2533090649.

47. Manual on Contrast Media. v.10.2. Reston, VA: American College of Radiology; 2013.

48. Ray JG, Vermeulen MJ, Bharatha A, Montanera WJ, Park AL. Association between MRI exposure during pregnancy and fetal and childhood outcomes. JAMA. 2016 Sep 6;316(9):952-961. doi: 10.1001/jama.2016.12126.

49. Semelka RC, Ramalho J, Vakharia A, et al. Gadolinium deposition disease: initial description of a disease that has been around for a while. Magn Reson Imaging. 2016 Dec;34(10):1383-1390. doi: 10.1016/j.mri.2016.07.016.

50. Burke LM, Ramalho M, AlObaidy M, Chang E, Jay M, Semelka RC. Self-reported gadolinium toxicity: a survey of patients with chronic symptoms. Magn Reson Imaging. 2016 Oct;34(8):1078-1080. doi: 10.1016/j.mri.2016.05.005.

51. Consortium of MS Centers MRI Protocol and Clinical Guidelines for the Diagnosis and Follow-up of MS: 2018 Revised Guidelines. Hackensack, NJ: Consortium of Multiple Sclerosis Centers; 2018.

52. Gulani V, Calamante F, Shellock FG, Kanal E, Reeder SB; International Society for Magnetic Resonance in Medicine. Gadolinium deposition in the brain: summary of evidence and recommendations. Lancet Neurol. 2017 Jul;16(7):564-570. doi: 10.1016/S1474-4422(17)30158-8.

53. Consortium of MS Centers MRI Protocol for the Diagnosis and Follow-up of MS. Hackensack, NJ: Consortium of Multiple Sclerosis Centers; 2017.

54. Gale EM, Atanasova IP, Blasi F, Ay I, Caravan P. A manganese alternative to gadolinium for MRI contrast. J Am Chem Soc. 2015 Dec 16;137(49):15548-15557. doi: 10.1021/jacs.5b10748.

55. McCullough BJ, Kolokythas O, Maki JH, Green DE. Ferumoxytol in clinical practice: implications for MRI. J Magn Reson Imaging. 2013 Jun;37(6):1476-1479. doi: 10.1002/jmri.23879.

56. Vasanawala SS, Nguyen KL, Hope MD, et al. Safety and technique of ferumoxytol administration for MRI. Magn Reson Med. 2016 May;75(5):2107-2111. doi: 10.1002/mrm.26151.

57. Rigby PJ. Comparison of FIESTA and gadolinium-enhanced T1-weighted sequences in magnetic resonance of acoustic schwannoma. The Radiographer. 2006;53(2):11-21.

58. Zidan DZ, Elghazaly HA. Can unenhanced multiparametric MRI substitute gadolinium-enhanced MRI in the characterization of vertebral marrow infiltrative lesions? Egyptian J Radiol Nucl Med. 2014;45(2):443-453. doi: 10.1016/j.ejrnm.2014.02.014.

This article meets the Accreditation Council for Graduate Medical Education and the American Board of Medical Specialties Maintenance of Certification competencies for Patient Care, Medical Knowledge, and Systems-Based Practice. 